\title{
CELL PHONE OPERATED CAR USING BLUETOOTH TECHNOLOGY AND ANDROID APPLICATION
}

\author{
Pooja Gangan ${ }^{1}$, Amol Joglekar ${ }^{2}$ \\ ${ }^{I}$ M.Sc Computer Science student, Mithibai College, Mumbai, Maharashtra, India \\ ${ }^{2}$ Guide and Professor, Computer Science, Mithibai College, Mumbai, Maharashtra, India
}

\section{INTRODUCTION}

Bluetooth is popular method of communication between devices. Many smart phones today, have the capability to communicate using Bluetooth. This is useful to mobile application developers whose applications require a wireless communication protocol.However, utilizing the Bluetooth API can be difficult for first time user. The objective of this proposed system is to explain how to use the Bluetooth tools available on Android and using instructions we can send and receive data to and from device wirelessly.

In principle, RF (Radio Frequency) emitted by Bluetooth can be regarded as the control which deals with the use of radio signal to remotely control any device. A remotely controlled car may be defined as any mobile device which is control by means that it does not restrict it motion with origin external to the device that is the possibility of an existence of a radio control device, a wireless medium between the Remote Mobile and Smart car. A Remote Car is always control by a human operator and it takes no positive action autonomously.

The control of smart car involves three distinct phases:

1) Perception

2) Processing

3) Action

The preceptors are sensors mounted on the robot, processing is done by the on-board microcontroller or processor, and the task (Action) is performed using motor or with some other actuators.

In order to operate the car, we need to send message to Bluetooth module. This message is send using BlueArc application which is installed on Remote Mobile.The BlueArc application is made using MIT App Inventor. The Bluetooth Technology is incorporated using a serial Bluetooth module H5-06 to create a connection between Arduino Uno R3 and Android Application. The HC-05 comes with a rich set of AT command set to perform various tasks such as changing the module's default settings including changing the pass code, the device name and the baud rate.

Here the Android Application acts as a Master and Bluetooth acts as a slave. The breadboard on which the circuit of Arduino and Bluetooth module interfacing is constructed is mounted on a steel chassis.
The output of Bluetooth module $\mathrm{HC}-05$ is given to the input port of Arduino Uno R3. The Arduino program for $\mathrm{HC}$ - 05 is feed in Arduino using Arduino IDE. This program then reads the output of the HC-05.

The smart car could either have a two wheel drive or a four wheel drive. This paper we proposed to have four wheel drives as it provides a better control and more torque than the two wheel system.

High torque Gear motors which are used for motion of the car are fixed to the bottom of the steel chassis, in the four wheel drive system which we have used in the configuration of our circuit; the motors on both the sides are managed independently of one another. However a single L239D motor driver IC is not enough to control the four motors and therefore paper suggests to have two L293D ICs to drive four motors. L293D is a typical Motor driver IC which allows DC gear to drive on either direction.L293D is a 16 pin IC which can control a set of two DC motors simultaneously in any direction; it means that you can control two motors using the concept of Dual H-Bridge.

$\mathrm{H}$-Bridge is a circuit which allows the voltage to be flown in either direction .In a single L239chip there are two H-Bridge circuit inside the IC which can rotate two DC motors independently. There are two enable pins on L293D-Pin 1 and Pin 9.For being able to drive the motor Pin 1 and Pin 9 need to be HIGH. For driving the motor with left H-Bridge we need to enable pin 1 to HIGH. And for right H-Bridge we need to make the pin 9 to $\mathrm{HIGH}$. If anyone of the either pin1 or pin 9 goes LOW then the motor in the corresponding section will suspend working. It's like a switch.

There are four input pins for this L293D. Pin 2 and 7 on the left and pin 15 and 10 on the right.

Left input pins will regulate the rotation of motor connected across left side and right input for motor on the right side. The motors are rotated on the basis of the inputs provided across the input as logic 0 or logic 1 .

The gear assembly helps in increasing the torque and reducing the speed. Using case statement it switches to the corresponding function, for forward movement both motor will rotate in the same direction and to move it back both the motor will rotate in opposite directions to that of forward 
direction. To move left and right either of the motor will rotate and to stop both motor will stop. Motors are given instructions, in the way user commands them through his phone.

\section{LITERATURE SURVEY}

In1898, Nikola Telsa built the first propeller-driven radio controlled boat, which can be regarded as the original prototype of all modern-day uninhabited aerial vehicles and precision guided weapons. Records state that it is the first among all remotely controlled vehicles in air, land or sea. It was powered by lead-acid batteries and an electric drive motor. The vessel was designed in such a way that it could be maneuvered alongside a target using instructions received from a wireless remote-control transmitter, Once in its position, a command would be sent to denote an explosive charge contained within the boat's forward compartment. The Weapon's guidance system introduced a secure communication link between the pilot's controller and the surface running torpedo in an effort to assure that control could be maintained even in the presence of electronic counter measures.

Wireless controlled unmanned vehicles which are used nowadays typically use RF circuits for motion and control. But RF circuits suffer from the disadvantage of limited working range which results in limited control .As RF circuits follows LOS(Line of sight) approach, it fails miserably in NLOS(Non-Line of sight) conditions involving obstacle and barriers. To overcome these, one method was proposed by Awab Farrah et al,(2010)[1] which typically makes use of the DTMF technology along with a microcontroller based circuit for maneuver and control of these unmanned robotic vehicles. Similarly, Ashish Yadav et al, (2012) [2] also proposed the construction of an unmanned vehicle which could be especially used for ground combat using a similar technology.

Recently, Sabuj Das Gupta et al (2013) [3] discussed in detail about how the method described in [1] could be implemented using a microcontroller by providing the necessary circuit details and the software code.

Hebah H.O.Nasereddin and Amjad Abdullah Abdelkarim[4] ,they have used the Bluetooth technology to control robot using Smartphone the same concept as I have mentioned but they have used Symbian Operating System which supports communication protocol and enables mobile phones to be a platform for development of application. In this proposed experiment robot is controlled using Bluetooth which has two modes: The first mode DDM called direct drive mode, in this case robot moves in all directions as per the user requirement the other mode is MBM called map based mode, in which MBA allows user to draw initial point and end point and obstacle, in order to calculate shortest path. So user can choose or use one of two modes to control robot using wireless communication.

Awab Fakih and Jovita Serrao[1], they have just shown that using two mobile phones and single microcontroller how the car moves or rotate in particular direction for that they have used DTMF technology. The concept is like one mobile phone is used as transmitter and other one is placed on robot as receiver which is set to 'auto answer mode'. So when there is call from transmitter; then after ring the cell phone which is on robot, accepts the call as it is on auto answer call mode after this when user press some key say 2 then the DTMF (Dual Tone Multiple Frequency) tone will be produced.

Then DTMF Decodes the received tone and sends that tone in binary format to microcontroller .According to program burn on microcontroller motor starts to rotate.

Ritika pahuja and Narender Kumar[5] stated the concept using HC-06 Bluetooth module with AT89S52 microcontroller in which they have burned the program code in C language where as I choose to implement in java because Arduino OS (ATmega328 microcontroller) is open source OS and platform independent. In short, while implementing proposed system there may be possibilities to make changes in code to meet the desired output so to burn program on ATmega328 i.e. Arduino Uno R3 is convenient way or we can say it allows to burn code whenever developer want to make changes using open source MIT App Inventor.

\section{RESEARCH METHODOLOGY}

\subsection{Block Diagram}

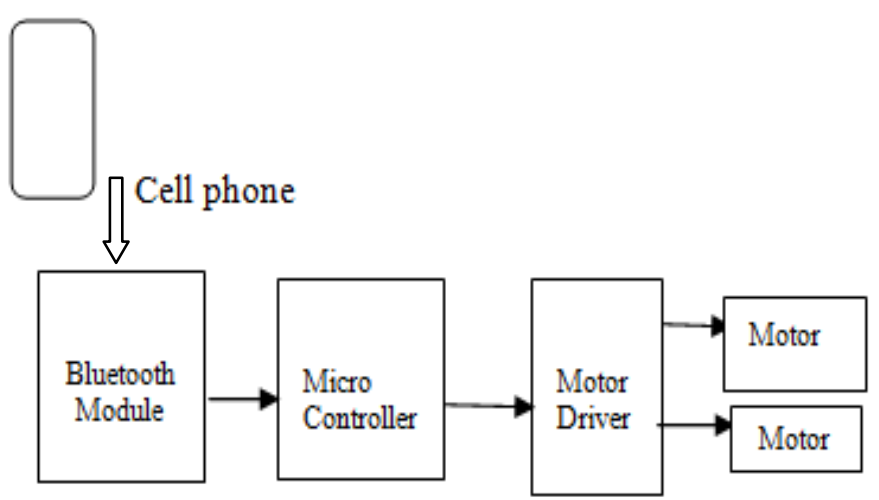

Fig 1: Block diagram of proposed system

Here Smartphone is used as remote mobile which operates the car using Android Application and Bluetooth module to send instruction to microcontroller. Bluetooth module is used for wireless communication between two devices or no of devices. The instructions from Bluetooth module microcontroller plays an important role and it sends its output to Motor driver so that motor can rotate and hence the car can be moved from one location to another.

\subsection{Flowchart}

Initial step of this is to pair HC-05 Bluetooth module with mobile. Once the connection is established we need to press connect so that it gets triggered with a default password. There will be a predefined lookup table coded in a program which contents the information of direction and ASCII code. 


\begin{tabular}{|l|l|}
\hline Direction & code \\
\hline FRONT & 1 \\
\hline LEFT & 2 \\
\hline RIGHT & 4 \\
\hline STOP & 3 \\
\hline
\end{tabular}

Fig 2: lookup table.

When user press "FRONT" icon it sends ASCII value of 1 to Bluetooth module which sends that value to microcontroller to check the equivalent code using lookup table. Once the value is detected the signal will be passed to a motor driver circuit. So it moves the car in forward direction.

When user press "LEFT" icon it sends ASCII value of 2 to Bluetooth module then it sends the signal to microcontroller and microcontroller will send the signal to motor driver to move the car in left direction for value 2. On press of "stop" icon generally it stops the working process. it sends ASCII value of 3 through Bluetooth module to microcontroller the microcontroller send it to motor drive to stop the motion of car.

When we press "RIGHT" icon it send ASCII value of 4 to Bluetooth module then it sends the signal to microcontroller and microcontroller will send the signal to motor driver to move the car in right direction.

Similarly, for press "REVERSE" icon it sends ASCII value of 5 to Bluetooth module then it sends the signal to microcontroller and microcontroller will send the signal to motor driver in order to move the car in reverse or backward direction.

Finally DISCONNECT \& CLOSE to disconnect paired Bluetooth module.

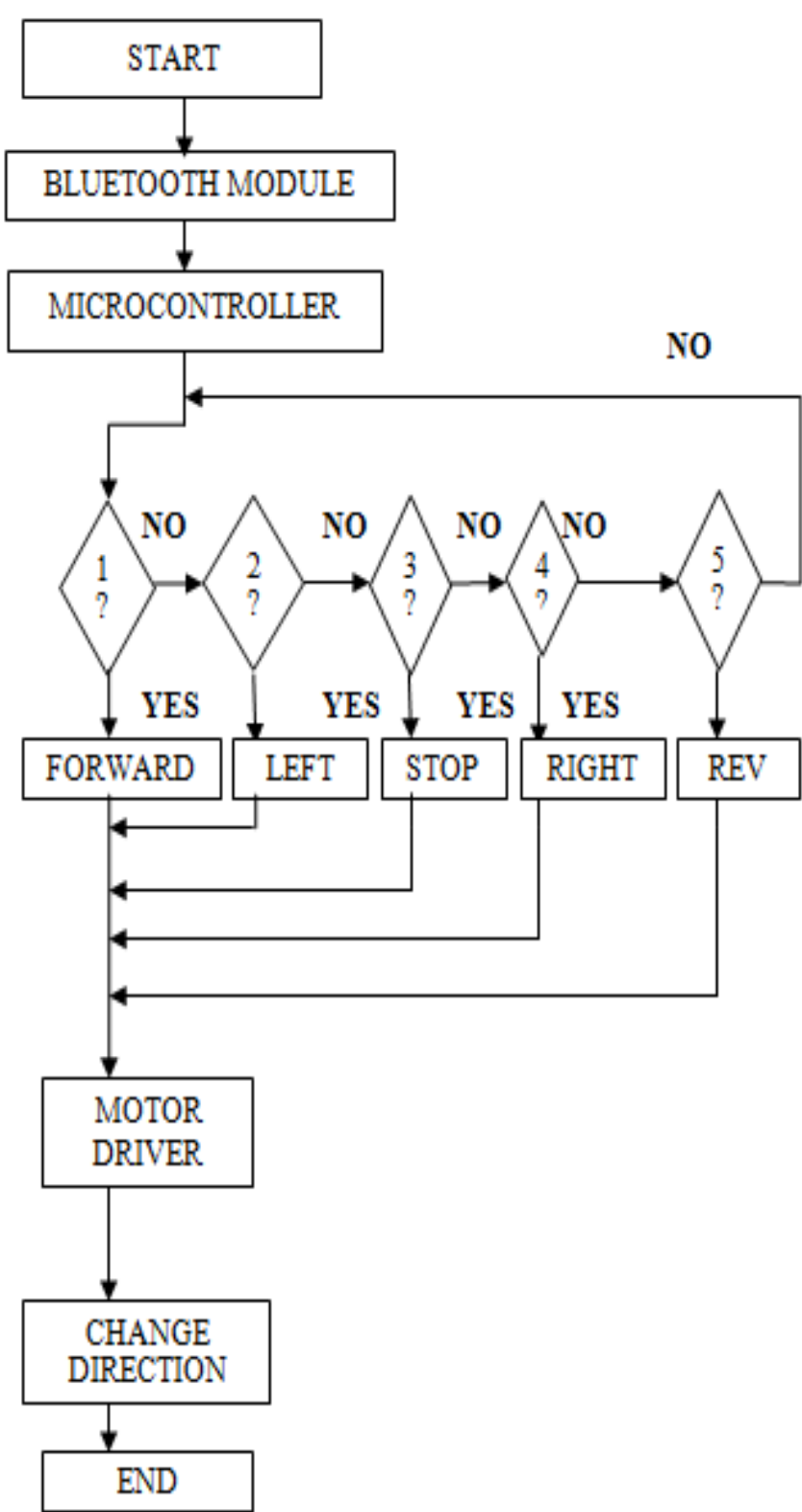

Fig 3: Flowchart of proposed system

\subsection{Proposed Algorithm}

Step 1: Establish Communication between Android and Bluetooth Module.

Step 2: Press Command input button from App.

Step 3: Corresponding Command input string is send to Bluetooth Module.

Step 4: Check Command is received by the Bluetooth module.

Step 5: If "Disconnect" Command given then end communication.

Step 6: Close Application.

\section{Algorithm for Arduino}

Step 1: If input is received, check for a string received.

Step 2: Compare contain of string received with pre-defined command in Arduino. 
Step 3: Command matches with desired if-else block and motor will drive accordingly to output of that block.

Step 4: If Command received is "Stop" entire process will stop.

Step 5 If Command received is "Disconnect" it will reset all the codes.

\section{FUTURE SCOPE}

We can modify the rover as per our requirement. If we want to use this for surveillance purpose so we can implement the camera or thermal sensors along with others sensors. Further if we want to improve the accuracy of the rover, we can implement sonar sensors so controlling rover from remote place can be possible. We can also implement GPS system so it can be semi-autonomous. The proposed work can be enhanced with the help of more security function like passwords and so on.

\section{CONCLUSION}

With the help of wireless technology in combination with Bluetooth we can create wonders and this paper proposes an idea of moving car. This technology can be useful for parking cars at big places like malls etc. and we can reduce man power to park cars.

\section{REFERENCES}

[1]. Awab Fakih, Jovita Serrao, "Cell Phone Operated Robotic Car" International Journal of Scientific and Engineering Research, ISSN 2229-551.

[2]. Gupta, Sabuj Das, Arman Riaz Ochi, Mohammad Sakib Hossain and Nahid Alam Siddiqi. "Designing \& Implementation of Mobile Operated Toy Car by DTMF" International Journal of Scientific \& Research Publications, Vol-3, Issue-1, 2013 ISSN 2250-3153.

[3]. Jadhav, Ashish, Mahesh Kumbhar and Meenakshi Pawar."Cell Phone Controlled Ground Combat Vehicle" International Journal of Computer and Communication Engineering, Vol.1, No.2, July2012.

[4]. Hebah H.O.Nasereddin \& Amjad Abdullah Abdelkarim, "SMARTPHONE CONTROL ROBOTS THROUGH BLUETOOTH" Middle East University, Amman, Jordan. Amman Arab University, Jordan.

[5]. Ritika Pahuja \& Narender Kumar, "Android Mobile Phone Controlled Bluetooth Robot Using 8051 Microcontroller." International Journal of Scientific Engineering and Research (IJSER), Volume 2 issue 7, July 2014.

[6]. Banerji,Sourangsu. "Design and Implementation of an Unmanned Vehicle using a GSM network with Microcontrollers" International Journal of Science, Engineering and Technology Research 2.2(2013): pp-367374.

[7]. Shashanka, D. "Password Protection for DTMF Controlled Systems without Using a Microcontroller." International Conference on Computing \& Control Engineering, 2012.

[8]. Edwin Wise, Robotics Demystified (Mc-GrawHill, 2005).
[9]. www.instructables.com [10]. www.jeeexplore.ieee.org

[11]. www.electronichub.org

[12]. www.iaarc.org 